\title{
Ectopic thyroid in duplicated gall bladder: a rare entity. Case report.
}

\author{
Manohar B. Kachare', Ashish Khandelwal' ${ }^{2}$, Sanjay B. Kulkarni ${ }^{3}$, Sachin S. Saboo ${ }^{2}$ \\ ${ }^{1}$ Department of Radiology, Govt. Medical College, Miraj, Maharastra, India \\ ${ }^{2}$ Department of Radiology, Brigham and Women's Hospital \& Harvard Medical School, Boston,USA \\ ${ }^{3}$ Department of Surgery, Vishrambag Hospital, Sangli, Maharastra, India
}

\begin{abstract}
Duplication of the gall bladder is a rare anomaly. Ectopic thyroid in a gall bladder is also exceedingly rare with only four cases of ectopic thyroid tissue in the gall bladder reported in English literature. We report the unique case of ectopic thyroid tissue in a duplicated gall bladder in a 51-year-old female with recurrent right upper quadrant pain. Abdominal sonography revealed a duplicated gall bladder with the cystic-solid mass in the septum separating the two gall bladders while subsequent histopathology from the cholecystectomy specimen confirmed ectopic thyroid tissue within the wall of double gall bladder.

Keywords: gall bladder duplication, thyroid gland, ectopic thyroid, ultrasonograph
\end{abstract}

\section{Introduction}

Ectopic thyroid which is defined as the presence of benign thyroid cells at sites other than anterior or lateral to the second, third, and fourth tracheal rings is a rare entity [1]. Gallbladder duplication is a rare entity, with reported autopsy incidence of about 1:4000 [2]. Thyroid develops at the base of tongue from endoderm and descends inferiorly below the level of cricoid cartilage anterior to the trachea. Base of tongue accounts for $90 \%$ of ectopic thyroid cases which is likely due to anomalous migration from its normal pathway [3]. However, ectopic thyroid below the diaphragm is difficult to explain which however occurs very rarely. To date, only four cases of ectopic thyroid in a gall bladder have been reported which were mostly detected in surgically resected specimens $[3,4]$. To our knowledge, this is the first case of ectopic thyroid in duplicated gall bladder.

Received 27.10.2012 Accepted 17.12.2012

Med Ultrason

2013, Vol. 15, No 1, 73-75

Corresponding author: Sachin S. Saboo, MD, FRCR

Brigham and Women's Hospital

Harvard Medical School

Boston, MA-02115, USA

Phone: 617-817-9353, Fax: 857-307-2011

Email: ssaboo@partners.org

\section{Case Report}

A 51-year-old female was referred for abdominal sonography for recurrent right upper quadrant abdominal pain but without any other significant history. Sonography (Sonoline Elegra, 3.5 MHz convex transducer; Siemens Medical Solutions USA, Inc., Malvern, Pa., USA) of the upper abdomen revealed duplication of gall bladder. Additional findings included $3 \times 1 \mathrm{~cm}$ mildly thick walled predominantly cystic lesion with hypoechoic peripheral nodular solid component within the neck region of septum between these two gall bladder moieties (fig 1). The walls of duplicated gall bladder were thickened with minimal pericholecystic fluid. The common hepatic, intrahepatic and extrahepatic bile ducts were normal in caliber. The possibility of duplicated gall bladder with acute cholecystitis was raised with indeterminate etiology of the lesion in the septum between the two moieties. In view of acute clinical findings, laparoscopic cholecystectomy was performed. Cholecystectomy gross specimen examination revealed longitudinal septum dividing the gallbladder cavity in two halves confirming duplication. Septum revealed $3 \times 2 \mathrm{~cm}$ cystic lesion filled with colloid like material and brownish areas in the wall. Microscopic examination revealed denuded mucosa with diffuse mononuclear leucocytic to lymphocytic inflam- 
mation in the wall with columnar epithelial lining and thyroid parenchyma in its wall (fig 2).

Hence ${ }^{99 m}$ Tc-DTPA scintigraphy scan was performed post-operatively to evaluate for ectopic thyroid tissue and exclude thyroid neoplasm, which however revealed normal tracer uptake in thyroid and salivary glands (fig 3); sonography of the thyroid gland did not reveal any neoplasm, thereby excluding metastatic lesion from thyroid gland in the GB fossa.

\section{Discussion}

Ectopic thyroid which is defined as the presence of benign thyroid cells at sites other than anterior or lateral to the second, third, and fourth tracheal rings is a rare entity [1]. Gallbladder duplication is a rare entity, with reported autopsy incidence of about 1:4000 [2]. Embryologically, thyroid gland develops from endodermal diverticulum at foramen caecum which then descends anterior to hyoid bone and laryngeal cartilages by seventh gestational week. Ectopic thyroid can occur at any site along its route of descent and rarely in locations other than its normal path of descent. Lingual thyroid is the most common location of ectopic thyroid accounting for $90 \%$ of cases, while rare sites in the neck include suprahyoid, infrahyoid, or subhyoid levels, in the remnants of thyroglossal duct, and submandibular regions $[3,4]$. Intrathoracic sites are very rare with known sites of involvement include the heart, ascending aorta, thymus, oesophagus, and lungs. Intra-abdominal ectopic location is extremely rare which include duodenum, gallbladder, pancreas, small bowel mesentry, porta hepatis, adrenal gland, ovary, fallopian tube, uterus and vagina [5].

To date, only four cases of ectopic thyroid in gall bladder have been s reported which were mostly detected in surgically resected specimen $[3,4]$. One case report described ectopic thyroid tissue in the gall bladder as a solid gall bladder wall mass on preoperative sonography [4]. However, occurrence of ectopic thyroid within or adjacent to the gallbladder is often an incidental finding in histopathology after cholecystectomy for acute or chronic cholecystitis [5]. The cause of the occurrence of ectopic thyroid tissue in the gall bladder is difficult to explain. However, possibility of aberrant migration or metaplastic or heteroplastic phenomenon may be responsible, since the proximal segment of the gastrointestinal tract, liver, ventral pancreas, and the thyroid all have a common embryologic origin from the foregut endoderm [5].

Our case is unique describing the association of two embryologically diverse entities of duplication of the gall bladder and ectopic thyroid tissue in a single pa-

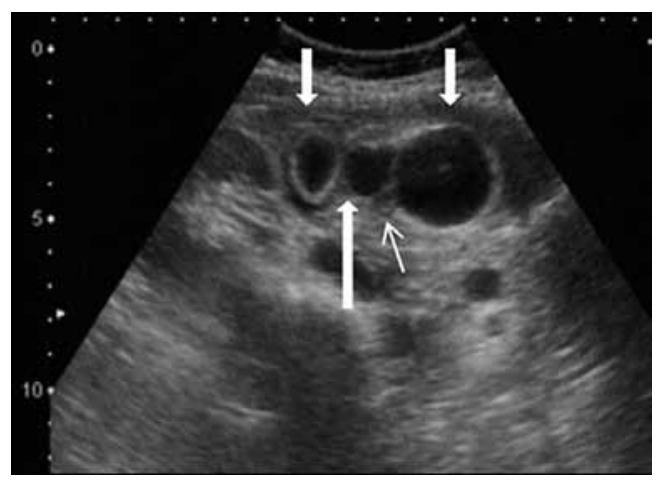

Fig 1. Transverse sonogram of the right upper quadrant of the abdomen reveals duplication of gall bladder with mildly thick wall (short arrow) and $3 \times 1 \mathrm{~cm}$ mildly thick walled predominantly cystic lesion (long arrow) containing a hypoechoic eccentric solid nodular component (thin arrow) within the neck region of septum between these two gall bladder moieties.

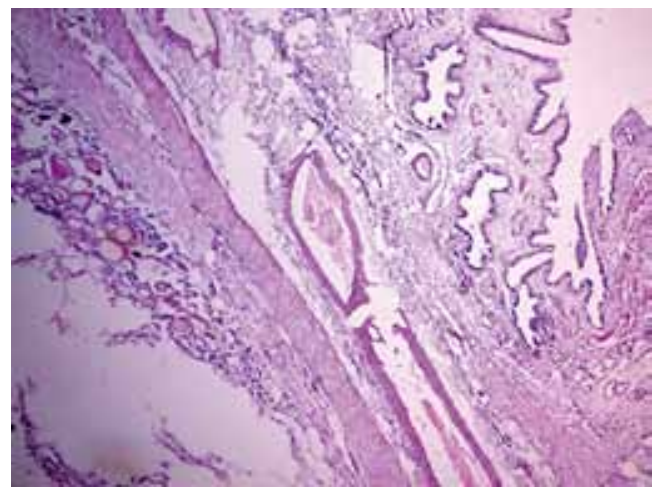

Fig 2. Histological examination with hematoxylineosin staining revealing denuded mucosa, columnar epithelial lining, and diffuse mononuclear leucocytic to lymphocytic inflammation with thyroid parenchyma in its wall.

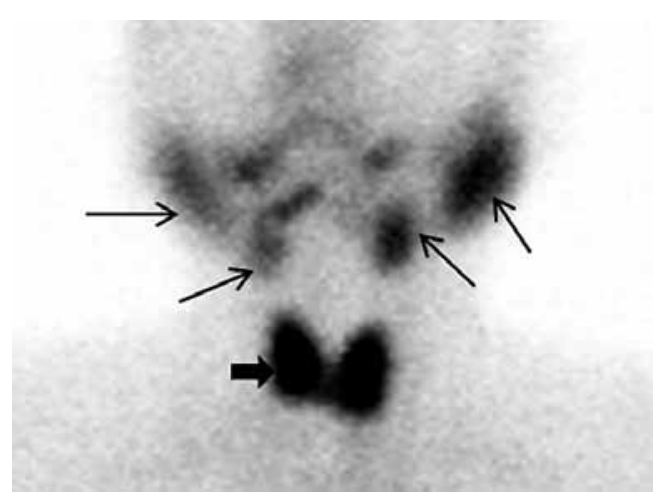

Fig 3. Post-operative $99 \mathrm{mTc}$-DTPA scan image of the anterior view of the neck shows a normal physiological tracer uptake in thyroid (short arrow), and salivary glands (long arrow) in this clinically euthyroid patient. 
tient which have never been described together. In addition, preoperative suspicion of the lesion was raised on sonography in a patient who presented clinically as acute cholecystitis. These patients may present with or without hyperthyroidism and scintigraphy can be of value in detecting the ectopic thyroid tissue focus as seen in the present scenario, even though it was performed postoperatively. However, as the ectopic thyroid tissues can potentially mimic neoplastic process in the gall bladder fossa, metastatic thyroid neoplasm should always be excluded before making the diagnosis of ectopic thyroid tissue [4].

In conclusion, we have described duplication of gall bladder with ectopic thyroid tissue as an entirely de novo association. Preoperative assessment is possible with imaging modalities. However, it is important to exclude neoplastic process whenever thyroid tissue is ectopic in location.

\section{References:}

1. Mussak E, Kacker A. Surgical and medical management of midline ectopic thyroid. Otolaryngol Head Neck Surg 2007; 136: 870-872.

2. Roldan-Valadez E, Osorio-Peralta S, Vivas-Bonilla I, Vazquez-LaMadrid J, Alva-Lopez LF, Hernandez-Ortiz J. Asymptomatic true gallbladder duplication: a case report and review of the literature. Acta Radiol 2004; 45: 810814.

3. Cassol CA, Noria D, Asa SL. Ectopic thyroid tissue within the gall bladder: case report and brief review of the literature. Endocr Pathol 2010; 21: 263-265.

4. Liang K, Liu JF, Wang YH, Tang GC, Teng LH, Li F. Ectopic thyroid presenting as a gallbladder mass. Ann R Coll Surg Engl 2010; 92: W4-6.

5. Noussios G, Anagnostis P, Goulis DG, Lappas D, Natsis K. Ectopic thyroid tissue: anatomical, clinical, and surgical implications of a rare entity. Eur J Endocrinol 2011; 165: 375-382. 